\title{
Common Language of New Era in Sport Clubs: Emojis
}

\author{
Arif Yüce', Volkan Aydoğdu' ${ }^{2}$, Hakan Katırcı ${ }^{3}$ \\ ${ }^{1}$ ORCID iD: 0000-0003-3756-3870, Eskişehir Technical University, Eskişehir \\ Teknik Üniversitesi 2 Eylül Kampüsü, 26555 Tepebaşı/Eskişehir, Turkey \\ ${ }^{2}$ ORCID iD: 0000-0001-6044-2618, Eskişehir Technical University, Eskişehir \\ Teknik Üniversitesi 2 Eylül Kampüsü, 26555 Tepebaşı/Eskişehir, Turkey \\ ${ }^{3}$ ORCID iD: 0000-0002-2337-7711, Eskișehir Technical University, Eskișehir \\ Teknik Üniversitesi 2 Eylül Kampüsü, 26555 Tepebaşı/Eskişehir, Turkey \\ *Corresponding author, e-mail: arifyuce@eskisehir.edu.tr
}

\begin{abstract}
Defined as an easy and automated way of expressing emotions in the digital age, emojis are emerging as a new language in the social media world and sports clubs also. For sports clubs, it is of vital importance to communicate and establish effective relations with fans or followers. Hence, almost all professional sports clubs use social media and shape their social media accounts to interact with fans/followers and effectively maintain marketing communication efforts. The aim of this study was to determine the content of emoji usage in tweets of Turkish sports clubs (Besiktas JK, Fenerbahce SK, Galatasaray SK, Istanbul Başakşehir FK, Trabzonspor SK). Since Twitter is one of the most heavily used social media networks of sports clubs, so in this study was preferred. Content analysis method was used to examine emojis used by sports clubs. The study found that sports clubs use emojis that create positive and neutral connotations. Emojis used are heavily determined to be visuals depicting the colors and symbols of sports clubs. The study is the first to examine sports clubs' emojis used. Hence, the study included important results for the management of communication and marketing strategies of sports clubs on social media.
\end{abstract}

Keywords: Content Analysis, Social Media, Twitter, Sports Clubs, Emoji.

\section{Introduction}

Today, industrial development and change experienced in parallel with the developing technology has affected communication processes and forms along with all other fields. The development of internet technologies, in particular, accelerated the flow of information, thus a transformation, in which the perception of time and space was restructured and partially lost its importance, was experienced (Bauer et al., 2005; Figge, 2004; Garris \& Mishra, 2014; Yengin, 2015). This transformation, called 'new media' or 'second media age', represents a quite large area. New media, which covers all of the new communication technologies, is defined as the media that direct the traditional media into digital data, facilitate access to large audiences, and provide production, distribution and sharing through computers (Manovich, 2001; Poster, 2013; Tocci et al., 2007).

In the second media age, social networks are among the leading media channels. Social networks have laid the groundwork for the transfer of communication and relationships related to human life to the virtual environment (Lefever, 2012). According to the We are Social Inc., (2019) report there are 3.5 billion active social media users all over the world. In this context, it can be stated that $42 \%$ of the world's population is social media users. These statistics indicate that social media networks are the favorite of the new communication age. In this aspect, social media has become an 
indispensable phenomenon of life, not only changing the ways of communication between individuals (Boulianne, 2015; Lin \& Lu, 2011; Maree, 2017) but also affecting all forms of communication such as B2C (Business to Customer]) and B2B (Business to Business). It can be considered that one of the consequences that this effect brings about is the emergence of a new form of written communication belonging to the digital world (Rodrigues et al., 2018). However, the inability of the language used online in this emerging form of communication to convey the desired emotions to the recipient has also led to the need for easier and faster communication (Hougaard \& Rathje, 2018; Stark \& Crawford, 2015; Zhou et al., 2017). This and similar situations caused users to create messages accompanied by visual and iconic messages, even accompanied by illustrated texts, to convey both emotional and conceptual messages, and thus, in this context, emojis have emerged as a new language in online communication (Gülşen, 2016; Cincu, 2017).

The word emoji (Cowie et al., 2001; McBain, 2017), denoted as an easy and automated way for individuals to express their emotions in the digital age, consists of the Japanese words 'e' meaning picture and 'moji' meaning 'letter, character' (Novak et al., 2015). The emergence of emojis occurred when Scott Fahlman added a smiley face ": -)" at the end of a sentence, which was likely to be misunderstood in an email he wrote in 1982, thinking this misunderstanding would be eliminated (Fahlman, 2002). After Fahlman, a collection of emoji was created in the late 20th century by Shigetaka Kurita using Japanese mangas and kanjis (Blagdon, 2013). The acceleration of computer-mediated communication means simultaneously has led to the development of thousands of emojis and the creation of a dictionary containing emoji meanings (Dresner \& Herring, 2010; Selken, 2019) The fact that Apple iPhone devices began to support emojis in 2010 is seen as one of the milestones in making emojis popular worldwide (Dimson, 2015). In forthcoming processes, the fact that the Oxford Dictionary first selected an emoji (face with tears of joy 0 ) as the word of the year (instead of a real word) in 2015 has enabled emojis to rise rapidly and take a different position in the digital world (Berard, 2018). In addition, Danesi, (2017) notes that another event that draws attention to the involvement of emojis in human life was revealed at a hearing held in New York City. During the trial, the judge instructed the jury that symbols (emoji) in the messages needs to be considered. More clearly, the court held that an emoji is an evidence to reveal the intended meaning, and therefore emojis should be regarded as explanatory elements like oral expressions (Danesi, 2017). Both events have characteristics that indicate that emojis are in the midst of everyday life.

It is argued that emojis, which are denoted as the alternatives of gestures and mimics used in face-to-face communication in electronic media, contribute to the meaning of written messages, strengthen the text and make them enjoyable (Highfield, 2018; Marengo et al., 2017; Pavalanathan \& Eisenstein, 2016; Pohl et al., 2017; Rezabek \& Cochenour, 1998; Swaney-Stueve et al., 2018). This power attributed to emojis not only enables interpersonal interaction in online communication but also enables brands in the marketing world to transfer their messages to consumers more creatively (Das et al., 2019; Jaeger et al., 2018; Stark \& Crawford, 2015). Studies (Dua, 2015; Kaye., 2015) indicate that emojis can make brands more recallable by adding energy to messages beyond their ordinary meaning.

This power of emojis has also attracted the attention of sports clubs within the sports world. For all sports organizations, especially sports clubs, it is of vital importance to communicate/interact and establish effective media relations with 
audiences, fans or followers (Dima, 2015; Grönroos, 2004; M.Nisar et al., 2018; Nicholson et al., 2015; Siguencia et al., 2017). At this point, it can be stated that successful management in the field of sports can be achieved by the correct management of the processes related to information and communication technologies. Studies (Abeza, G. et al., 2017; Filo et al., 2015; Gibbs, C., \& Haynes, 2013; Gibbs et al., 2014; M.Nisar et al., 2018; O'Shea; \& Alonso, 2011; Siguencia et al., 2017; Wang \& Zhou, 2015) reveal that almost all professional sports clubs actively use social media and shape their social media accounts within the scope of basic purposes, such as interacting with fans/followers, effectively performing and maintaining marketing communication efforts. Similarly, it is stated that sports clubs that interact with their fans and followers over the internet also use social media and emojis as a communication point, thus making the messages they want to convey to their target audience more effective (Blaszka, 2011). Some studies (Price et al., 2013; Watkins, 2013; Williams \& Chinn, 2010), on the other hand, have found that social media plays an important role in communicating with passionate young fans, that fans are becoming more interested in teams and leagues thanks to social media, and that fans use social media to both enhance and satisfy their social and supporter identities. Another important point revealed in the same studies is the conclusion that social media forms a strong bond between fans and the club. Twitter is one of the most heavily used social media networks of sports clubs (Wang \& Zhou, 2015).

According to Grewal \& Levy, (2019), social media can be classified into three categories as social network sites (e.g. Facebook, Twitter), media-sharing sites (e.g. Youtube), and thought-sharing sites (e.g. Blogs). Although all social media networks are often used depending on user preferences, Twitter has gained the status of being frequently preferred by sports organizations, sports clubs, and sports fans (Wang, 2021; Winand et al., 2019; Zanini et al., 2019). Also, Twitter is used by sports organizations as a much more direct and dialogue-based communication tool with fans as part of their branding efforts (Pegoraro, 2010). In addition to these, Twitter differs from other social media networks based on creating micro-blogs among social media types and crossdistribution feature of the application due to its growing user base, allowing it to reach out to and communicate with more users (Price et al., 2013). In other words, the Twitter platform enables interaction along with real-time updates that enable a brand to reflect its mission and vision, communicate with followers in different ways (Hambrick, 2012; Hopkins, 2013).

The sports clubs competing in major football leagues (e.g. England Premier League, Bundesliga, La Liga) include emojis that have a common meaning and are accepted by everyone in their posts to reach their fans who speak different languages in different countries-continents ( $\mathrm{Li}$ et al., 2020). A study conducted on the use of Twitter by sports clubs in Germany found that sports clubs often use the Twitter platform for three different purposes: providing and distributing information directly to and communicating with their fans (Oelrichs, 2020). It was revealed that Beşiktaş, Fenerbahçe, and Galatasaray sports clubs in Turkey share an average of 11.3 tweets per day via the Twitter platform, while Benfica, Porto and Sporting in Portugal make an average of 19.3 tweets per day (Drapier \& Tezonarici, 2019). In this respect, the sports clubs have been carrying out their public relations activities and similar announcements, mainly their transfer disclosures and promotion campaigns, via Twitter, especially in 2017 and thereafter. Also, the emojis that will arouse excitement, curiosity, and interest in fans and followers have been used in these posts. 
According to 2019 data, among the three football clubs with the highest number of followers on the Twitter platform are Real Madrid with 32.6 million followers, FC Barcelona with 30.3 million followers, and Manchester United with 19.8 million followers (Tweetfc, 2019). All the information and relevant figures reveal that Twitter can create unique communication opportunities with its various aspects on behalf of sports clubs. Because of all these reasons, in this study, the Twitter platform was preferred in order to get the communication styles and preferences of sports clubs with their fans-followers on social media networks in a more detailed and more realistic manner and in their own context. In the light of this information, the aim of this study is to examine the emoji usage patterns and content with the content analysis method of Turkish sports clubs on Twitter platform, which is one of the social media networks that can be considered an indispensable element of the digital age and where fans and sports clubs communicate intensively.

In the context of communication and strategic communication in sports, there are no previous studies on the use and content of emojis in posts that sports clubs make on social media networks. Accordingly, this study is the first in the relevant field. Although this is considered one of the most important original values of the study, this study contains important clues about how fan-sports club interaction occurs in the context of emojis through the Twitter platform, which is one of the social media networks. For all these reasons, this study has the qualities to make an important and original contribution to sports and communication literature.

\section{Methods}

Within the scope of the study, official Twitter accounts, which are one of the active social media tools used by sports clubs in Turkey, and emojis used in their posts through these accounts were examined using the content analysis method from qualitative research techniques. Content analysis is one of the most important research techniques used in the social sciences, especially in communication texts (Fraenkel et al., 2012; Neuman, 2020). Content analysis, defined basically as the analysis of the written contents of a communication, is a method used to reduce many text words to fewer content categories, based on open coding rules (Krippendorff, 2004; Weber, 1990). The purposive sampling method was used in the study. In the purposeful sampling method, researchers are able to incorporate the group that they think it may represent the universe into their study by using their knowledge and experience. Accordingly, the official Twitter accounts of Istanbul Başakşehir FK (BŞK), Besiktas JK (BJK), Fenerbahce SK (FB), Galatasaray SK (GS) and Trabzonspor SK (TS) clubs, which are among the leading sports clubs in Turkey finishing in the top 6 places in the Turkish Football Super League between 2017 and 2019, indicated in Table 1, are included in the sample.

Table 1. Sports Clubs consisting of sample and their Official Twitter Accounts

\begin{tabular}{cc}
\hline Name of Sports Club & Official Twitter Account \\
\hline İstanbul Başakşehir FK & https://twitter.com/ibfk2014 \\
Beşiktaş JK & https://twitter.com/Besiktas \\
Fenerbahçe SK & https://twitter.com/Fenerbahce \\
Galatasaray SK & https://twitter.com/GalatasaraySK \\
Trabzonspor SK & https://twitter.com/Trabzonspor \\
\hline
\end{tabular}

Note: the official Twitter accounts of the five Sports Clubs 
Within the scope of the study, all posts (a total of 18.166 tweets) made between 22 January 2018 and 24 May 2019 from the official Twitter accounts of the sports clubs in the sample group were recorded. Then those tweets and the emojis used in the tweets were encoded by three PhD-level coders. Coders were trained by researchers according to the guiding principles proposed by Kolpe \& Burnett, (1991). Although there is little consensus in the field paper on the value of best encoder reliability (Lombard et al., 2006; Potter \& Levine-Donnerstein, 2009) the value of Krippendorff Alpha (Krippendorff, 2004) is used in study. In this context, the value of reliability between encoders is determined as (.98) in the study. This value indicates that the reliability value between encoders is appropriate for the interpretation of emojis used in tweets.

\section{Results}

The numerical data related to the posts and emojis posted in the official Twitter accounts of the sports clubs examined within the scope of the study are tabulated and provided in sequence.

Table 2. Numerical data on Twitter posts of sports clubs

\begin{tabular}{|c|c|c|c|c|}
\hline Season/Period & Sports Clubs & $\begin{array}{l}\text { Number of } \\
\text { Posts } \\
\text { (Tweets) } \\
\text { (n) }\end{array}$ & $\begin{array}{l}\text { Number of Emojis } \\
\text { within the Tweets } \\
\text { Including Emojis } \\
\text { (n) }\end{array}$ & $\begin{array}{l}\text { Average } \\
\text { Number of } \\
\text { Emoji per } \\
\text { Tweet } \\
(\bar{x})\end{array}$ \\
\hline $2017-2018$ & Başakşehir FK & 538 & 899 & 1.67 \\
\hline Season & Beşiktaş JK & 1418 & 743 & 0.52 \\
\hline $2^{\text {nd }}$ Period & Fenerbahçe SK & 1744 & 613 & 0.42 \\
\hline (22.01.2018- & Galatasaray SK & 1748 & 1009 & 0.57 \\
\hline 19.05.2018) & Trabzonspor SK & 793 & 468 & 0.59 \\
\hline & Total & 6.241 & 3.868 & 0.61 \\
\hline 2018-2019 & Başakşehir FK & 749 & 1331 & 1.77 \\
\hline Season & Beşiktaş JK & 1597 & 1331 & 0.83 \\
\hline $1^{\text {st }}$ Period & Fenerbahçe SK & 1224 & 1183 & 0.96 \\
\hline (10.08.2018- & Galatasaray SK & 1864 & 1816 & 0.97 \\
\hline 23.12.2018) & Trabzonspor SK & 868 & 603 & 0.69 \\
\hline & Total & 6.302 & 6.264 & 0.99 \\
\hline 2018-2019 & Başakşehir FK & 712 & 1306 & 1.83 \\
\hline Season & Beşiktaş JK & 1061 & 1001 & 0.94 \\
\hline $2^{\text {nd }}$ Period & Fenerbahçe SK & 1014 & 749 & 0.60 \\
\hline (19.01.2019- & Galatasaray SK & 2044 & 2136 & 1.04 \\
\hline 24.05.2019) & Trabzonspor SK & 792 & 598 & 0.75 \\
\hline & Total & 5.623 & 5.654 & 1.00 \\
\hline \multicolumn{2}{|c|}{ Grand Total } & 18.166 & 15.786 & 0.86 \\
\hline
\end{tabular}

Note: $\overline{\mathrm{x}}$ (mean), $\mathrm{n}$ (sample size)

As shown in Table 2, it is determined that a total of 18.166 tweets were examined during the period when the study was carried out and a total of 15.786 emojis were in these tweets. In the 2nd period of the 2017-2018 season, sports clubs posted a total of 6.241 tweets, while the total number of emojis in the tweets was determined to be 3.868. 
In the second period of the 2017-2018 season, it is determined that the club using the fewest emoji per tweet $(\bar{x}=1.67)$ is Fenerbahçe $S K$, while the club using the most emoji per tweet $(\bar{x}=0.42)$ is Başakşehir FK. In the first period of the 2018-2019 season, the total number of emojis used is 6.264, while the sports clubs post a total of 6.302 tweets. In the first period of the 2018-2019 season, it is determined that the club using the fewest emoji per tweet $(\overline{\mathrm{x}}=1.77)$ is Trabzonspor SK, while the club using the most emoji per tweet $(\bar{x}=0.69)$ is Başakşehir FK. In the second half of the 2018-2019 season, the total number of tweets posted is 5.623, while the number of emojis is 5.654. As in other seasons, in the second period of the 2018-2019 season, it is determined that the club using the most emoji per tweet $(\overline{\mathrm{x}}=1.83)$ is Başakşehir FK. Information about emojis on tweets of the sports clubs examined within the scope of the study is presented in tables.

Table 3. Content and numbers of emojis in the tweets of Başakşehir FK

\begin{tabular}{|c|c|c|c|c|c|c|c|c|c|c|c|c|c|c|}
\hline $\begin{array}{c}\text { Season/ } \\
\text { Period }\end{array}$ & $\begin{array}{l}: \frac{7}{\circ} \\
\text { 产 }\end{array}$ & 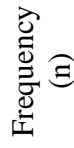 & 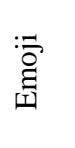 & 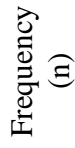 & 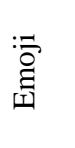 & 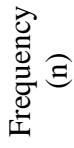 & $\begin{array}{l}:=\overline{0} \\
\text { 島 }\end{array}$ & 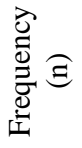 & 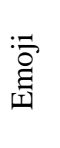 & 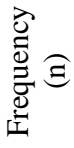 & 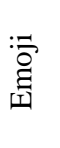 & 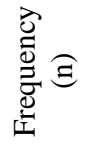 & 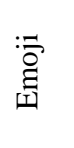 & 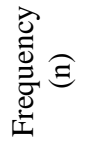 \\
\hline \multirow{13}{*}{$\begin{array}{l}2017-2018 \\
\text { Season } \\
2^{\text {nd }} \text { Period }\end{array}$} & $\infty$ & 216 & i & 13 & $\widehat{9}$ & 8 & $\rightleftarrows$ & 3 & $\Rightarrow$ & 2 & $\mathrm{i}$ & 1 & 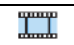 & 1 \\
\hline & 5 & 95 & 3 & 12 & 9 & 7 & $\alpha$ & 3 & 垔 & 2 & $\Rightarrow$ & 1 & $\boldsymbol{Q}$ & 1 \\
\hline & $\because$ & 47 & 4 & 12 & $\frac{1}{\mathrm{VS}}$ & 7 & 8 & 3 & 3 & 2 & 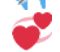 & 1 & $\because 0$ & 1 \\
\hline & 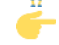 & 38 & $\checkmark$ & 12 & 事 & 6 & $\square$ & 3 & 5 & 2 & (l) & 1 & हु잉 & 1 \\
\hline & (1) & 37 & $\theta$ & 11 & $\ddot{\circ}$ & 6 & 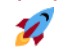 & 3 & 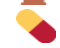 & 2 & 100 & 1 & $\because \theta$ & 1 \\
\hline & $\widehat{\lambda}$ & 29 & Q & 11 & d & 5 & 真 & 2 & $\Omega$ & 1 & 众 & 1 & by & 1 \\
\hline & Ø̈ & 28 & 7 & 10 & 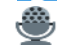 & 5 & 0 & 2 & 9 & 1 & $\tilde{x}$ & 1 & $\because$ & 1 \\
\hline & (1) & 28 & $(4)$ & 9 & $\frac{z_{z z}}{6}$ & 5 & 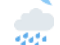 & 2 & 米 & 1 & b & 1 & $\Leftrightarrow$ & 1 \\
\hline & $\square$ & 22 & - & 9 & Q & 4 & $\frac{m a n}{21}$ & 2 & 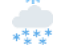 & 1 & $\sqrt{d}$ & 1 & & \\
\hline & $\vec{c}$ & 22 & $\Delta$ & 9 & $\forall$ & 4 & 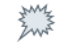 & 2 & $=$ & 1 & Jjת & 1 & & \\
\hline & 疋 & 21 & $\nabla$ & 9 & \%o & 4 & $\because 8$ & 2 & $\mathbb{N}$ & 1 & $\therefore$ & 1 & & \\
\hline & แற๊ & 20 & InI & 9 & $x$ & 4 & 8 & 2 & $=$ & 1 & $N$ & 1 & & \\
\hline & 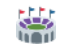 & 18 & (1) & 9 & $\cdots$ & 3 & $\%$ & 2 & $\therefore$ & 1 & 60 & 1 & & \\
\hline \multirow{24}{*}{$\begin{array}{c}2018-2019 \\
\text { Season } \\
1^{\text {st }} \text { Period }\end{array}$} & 5 & 236 & 6 & 13 & 算 & 5 & 75 & 3 & 算 & 1 & $\pi$ & 1 & 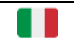 & 1 \\
\hline & $\Rightarrow$ & 163 & ist & 12 & 0 & 5 & (l) & 3 & 2 & 1 & $=$ & 1 & ㅁ & 1 \\
\hline & 0 & 114 & $\therefore$ & 12 & $d$ & 5 & 0 & 2 & 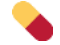 & 1 & $\ddot{\circ}$ & 1 & 8 & 1 \\
\hline & (2) & 93 & $\theta$ & 11 & Q & 5 & $\cdots$ & 2 & 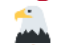 & 1 & $\because$. & 1 & $\%$ & 1 \\
\hline & 4 & 75 & 衫 & 11 & (1) & 5 & ? & 2 & 3 & 1 & W & 1 & 0 & 1 \\
\hline & $x^{\prime}$ & 58 & 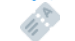 & 11 & ii & 4 & $\equiv \overline{Y S}$ & 2 & 4 & 1 & 8 & 1 & 9 & 1 \\
\hline & $\stackrel{n}{\oplus}$ & 51 & $\stackrel{z^{2} z}{0}$ & 10 & 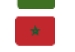 & 4 & 71 & 2 & 8 & 1 & 0 & 1 & 8 & 1 \\
\hline & $t$ & 34 & $\sqrt{ }$ & 10 & $\infty$ & 4 & 0 & 2 & $\theta$ & 1 & 至 & 1 & A & 1 \\
\hline & (3) & 31 & $\%$ & 8 & $\Leftrightarrow$ & 3 & 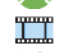 & 2 & $\because$ & 1 & 4 & 1 & 훌 & 1 \\
\hline & 5 & 26 & 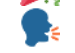 & 8 & 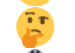 & 3 & 0 & 2 & 8 & 1 & 2 & 1 & .0 & 1 \\
\hline & $\overline{\mathrm{vS}}$ & 25 & (iii) & 8 & $\underline{\underline{3}}$ & 3 & 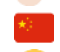 & 2 & $\frac{m a n d}{21}$ & 1 & $\theta$ & 1 & 䊀 & 1 \\
\hline & (D) & 23 & 鄇 & 8 & 8 & 3 & $\hat{*}$ & 2 & 3 & 1 & 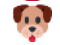 & 1 & 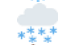 & 1 \\
\hline & ப̆ & 23 & H. & 8 & 晋 & 3 & II & 2 & 8 & 1 & 0 & 1 & 息 & 1 \\
\hline & 㗼 & 21 & 4 & 7 & 回 & 3 & 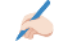 & 1 & 米 & 1 & 0 & 1 & $\mathscr{Q}$ & 1 \\
\hline & (N) & 20 & $\widehat{g}$ & 7 & $\mathbb{N}$ & 3 & 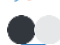 & 1 & $\therefore$ & 1 & $\pi$ & 1 & $\because$ & 1 \\
\hline & $\widehat{\lambda}^{\prime \prime \prime}$ & 18 & $Q$ & 7 & 뜨 & 3 & I & 1 & 를 & 1 & 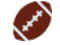 & 1 & 3 & 1 \\
\hline & c. & 17 & $Q$ & 6 & 2 & 3 & 0 & 1 & 11 & 1 & te & 1 & & \\
\hline & 5 & 285 & C. & 17 & 71 & 7 & A & 2 & 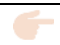 & 1 & 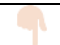 & 1 & $\tilde{\pi}$ & 1 \\
\hline & $\Rightarrow$ & 122 & (J) & 17 & ह & 5 & 4 & 2 & 무 & 1 & 米 & 1 & है & 1 \\
\hline & 0 & 103 & 4 & 16 & 粎炎 & 4 & $\because$ & 2 & $=$ & 1 & 0 & 1 & 시 & 1 \\
\hline & $\leftrightarrow$ & 72 & 2 & 15 & Ht & 4 & $M$ & 2 & $N$ & 1 & (5) & 1 & 8 & 1 \\
\hline & (2) & 64 & $\checkmark$ & 12 & $\theta$ & 3 & $\mathrm{i}$ & 2 & 18 & 1 & $\Leftrightarrow$ & 1 & 9 & 1 \\
\hline & $x$ & 50 & (1iv) & 12 & $\Leftrightarrow$ & 3 & $\sqrt{d}$ & 2 & II & 1 & î. & 1 & $\mathbb{N}$ & 1 \\
\hline & 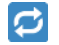 & 47 & $\stackrel{z^{2} z}{0}$ & 11 & $\frac{m_{n}^{m}}{m^{3}}$ & 3 & " & 2 & at & 1 & $=$ & 1 & 里 & 1 \\
\hline
\end{tabular}

Jurnal The Messenger, Vol. 13, No. 1 (2021), pp. 63-80 


\begin{tabular}{|c|c|c|c|c|c|c|c|c|c|c|c|c|c|c|}
\hline & & 45 & $\Leftrightarrow$ & 10 & - & 3 & $\because$ & 2 & 0 & 1 & 드. & 1 & 㸴 & 1 \\
\hline 2018-2019 & 도 & 37 & 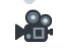 & 10 & 를 & 3 & & 2 & sys & 1 & 0 & 1 & & \\
\hline Season & VS & 31 & 3 & 9 & $\bullet$ & 3 & & 2 & 単 & 1 & $\xi$ & 1 & & \\
\hline $2^{\text {nd }}$ Period & 4 & 27 & $\ldots \%$ & 8 & 0 & 2 & $v_{2}$ & 2 & & 1 & 2 & 1 & & \\
\hline & $\boldsymbol{v}$ & 26 & 解 & 7 & $=\bar{\Omega}$ & 2 & $\cdots$ & 2 & & 1 & 绾 & 1 & & \\
\hline & $=$ & 20 & $\beta$ & 7 & 回 & 2 & 0 & 1 & 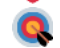 & 1 & $\approx$ & 1 & & \\
\hline & c & 19 & ev & 7 & 0 & 2 & 1 & 1 & ii: & 1 & wo & 1 & & \\
\hline & 訾 & 19 & 务 & 7 & 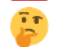 & 2 & 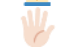 & 1 & 5 & 1 & $\because$ & 1 & & \\
\hline & 0 & 19 & Q & 7 & $\mathbf{Q}$ & 2 & : & 1 & 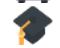 & 1 & 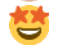 & 1 & & \\
\hline & 0 & 18 & 2 & 7 & 0 & 2 & 0 & 1 & 6 & 1 & (3)) & 1 & & \\
\hline
\end{tabular}

Note: Source from twitter.com/ibfk2014

As seen in Table 3, Başakşehir FK sed 86 different emoji in the second period of the 2017-2018 season, 118 in the first period of the 2018-2019 season, and 110 in the second period of the 2018-2019 season. It is determined that in these periods the most preferred emojis are soccer ball $(\mathrm{n}=616)$, geometric shape representing the club's colors $(\mathrm{n}=363)$, right arrow $\rightarrow(\mathrm{n}=285)$, camera with flash $(\mathrm{n}=254)$ and o'clock ( $\mathrm{n}=157)$.

Table 4. Content and numbers of emojis in the tweets of Beșiktaș JK

\begin{tabular}{|c|c|c|c|c|c|c|c|c|c|c|c|c|c|c|}
\hline $\begin{array}{c}\text { Season/ } \\
\text { Period }\end{array}$ & $\begin{array}{l}: \overline{0} \\
\stackrel{\overline{!}}{4}\end{array}$ & 离 & 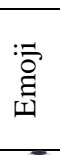 & 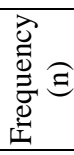 & $\begin{array}{l}:-\bar{\Xi} \\
\stackrel{1}{1}\end{array}$ & 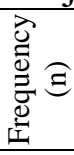 & $\begin{array}{l}\text { : } \\
\text { 童 }\end{array}$ & 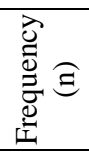 & 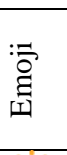 & 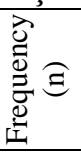 & $\begin{array}{l}: \overline{0} \\
\text { 息 }\end{array}$ & 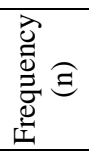 & $\begin{array}{l}: \overline{0} \\
\stackrel{\bar{\varepsilon}}{1}\end{array}$ & 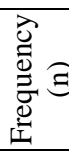 \\
\hline & 1 & 112 & 5 & 20 & 0 & 12 & $\ddot{\circ}$ & 7 & 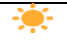 & 4 & 㩊 & 2 & 8 & 1 \\
\hline & N & 84 & 尚 & 19 & 0 & 11 & 3 & 7 & viv & 4 & 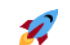 & 2 & & 1 \\
\hline & m & 73 & VS & 19 & $=$ & 11 & 些 & 4 & E & 4 & $\ddot{\otimes}$ & 2 & & 1 \\
\hline 2017-2018 & S & 50 & $?$ & 19 & $\Rightarrow$ & 9 & c. & 5 & d & 4 & 象 & 1 & & 1 \\
\hline Season & $\checkmark$ & 35 & (1) & 19 & 21 & 8 & 4 & 6 & דiע & 3 & 2 & 1 & 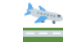 & 1 \\
\hline \multirow[t]{11}{*}{$2^{\text {nd }}$ Period } & 5 & 34 & 0 & 19 & 口 & 8 & (3) & 5 & 补 & 3 & ? & 1 & & \\
\hline & $\theta$ & 27 & at & 14 & 近 & 8 & III & 4 & $\cdots$ & 3 & 5 & 1 & & \\
\hline & $\sigma$ & 27 & 7 & 13 & $\theta$ & 7 & Ф & 4 & 2 & 2 & $n$ & 1 & & \\
\hline & w. & 193 & $\mathrm{~F}=$ & 21 & 1 & 5 & $\sigma^{7}$ & 2 & $\bullet$ & 1 & it & 1 & $\theta$ & 1 \\
\hline & $\rightarrow$ & 109 & 3 & 21 & 2 & 5 & te & 2 & m & 1 & $y_{y}$ & 1 & $\sqrt{4}$ & 1 \\
\hline & 6 & 79 & $m$ & 20 & 3 & 5 & 4 & 2 & Q & 1 & A & 1 & - & 1 \\
\hline & 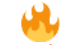 & 64 & 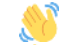 & 20 & บบำ & 5 & $\mathscr{P}$ & 2 & $\leq$ & 1 & 蒠 & 1 & $y$ & 1 \\
\hline & 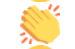 & 59 & E & 17 & 踣 & 5 & $\square$ & 2 & . & 1 & I+1 & 1 & $\gamma$ & 1 \\
\hline & $\theta$ & 54 & 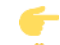 & 17 & $\because$ & 4 & : & 2 & 8 & 1 & $=$ & 1 & t & 1 \\
\hline & 5 & 50 & $a_{2}$ & 14 & ii & 4 & 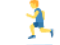 & 2 & so & 1 & a & 1 & 3 & 1 \\
\hline & $F$ & 49 & $\overrightarrow{\nabla t}$ & 14 & ; & 4 & 8 & 2 & 1 & 1 & $=$ & 1 & 6 & 1 \\
\hline \multirow{16}{*}{$\begin{array}{l}2018-2019 \\
\text { Season } \\
1^{\text {st }} \text { Period }\end{array}$} & $\checkmark$ & 37 & 10 & 12 & 来类 & 3 & $=$ & 2 & 8 & 1 & (i) & 1 & Het & 1 \\
\hline & e & 32 & 弯 & 12 & क्त? & 3 & SSIS & 2 & \% & 1 & $\vec{x}$ & 1 & 0 & 1 \\
\hline & VS & 28 & a & 9 & 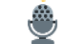 & 3 & 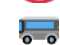 & 1 & $\Omega$ & 1 & $\because$ & 1 & 唃 & 1 \\
\hline & $\infty$ & 27 & $\theta$ & 8 & 1 & 3 & $\cdots$ & 1 & $\Omega$ & 1 & $\approx$ & 1 & A & 1 \\
\hline & 1 & 27 & 钧 & 8 & $\because 0^{\circ}$ & 3 & 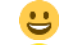 & 1 & 웅 & 1 & 0 & 1 & & 1 \\
\hline & 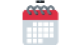 & 27 & 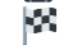 & 7 & 10 & 3 & 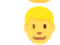 & 1 & (1) & 1 & $7^{18}$ & 1 & & \\
\hline & (1) & 27 & T & 6 & E & 3 & 柆 & 1 & 道 & 1 & 0 & 1 & & \\
\hline & c. & 24 & $\checkmark$ & 6 & 垔 & 3 & 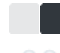 & 1 & 当 & 1 & $N$ & 1 & & \\
\hline & 口̆ & 23 & 泝 & 6 & $\alpha$ & 3 & $\cdots$ & 1 & $r .7$ & 1 & 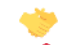 & 1 & & \\
\hline & $\Rightarrow$ & 22 & 16. & 6 & $\Leftrightarrow$ & 2 & 일 & 1 & 0 & 1 & 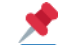 & 1 & & \\
\hline & $T$ & 22 & $\%$ & 5 & (11) & 2 & 2 & 1 & 'o" & 1 & 4 & 1 & & \\
\hline & in. & 255 & (1) & 17 & 5 & 7 & $\theta$ & 4 & 49 & 2 & 0 & 1 & $N$ & 1 \\
\hline & 5 & 65 & $\$$ & 16 & d, & 7 & Q & 4 & 善 & 1 & 需 & 1 & 89 & 1 \\
\hline & 5 & 36 & $\infty$ & 16 & $\Rightarrow$ & 7 & $\stackrel{\Omega}{\Omega}$ & 4 & 8 & 1 & in & 1 & 정 & 1 \\
\hline & $\mathbf{m}$ & 34 & VS & 16 & 0 & 6 & 8 & 4 & 11 & 1 & & 1 & $\bar{I}$ & 1 \\
\hline & 0 & 31 & ? & 16 & $\bullet$ & 6 & 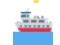 & 3 & $\theta$ & 1 & & 1 & T/ & 1 \\
\hline
\end{tabular}




\begin{tabular}{|c|c|c|c|c|c|c|c|c|c|c|c|c|c|c|}
\hline & 0 & 29 & $=$ & 16 & $\theta$ & 6 & 1 & 3 & . & 1 & () & 1 & $\Leftrightarrow$ & 1 \\
\hline 2018-2019 & (L) & 24 & 1 & 16 & $\%$ & 5 & 2 & 3 & $c$ & 1 & 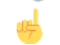 & 1 & e & 1 \\
\hline Season & $\theta$ & 24 & m & 14 & (1iv) & 5 & 些 & 3 & $=$ & 1 & 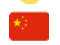 & 1 & coot & 1 \\
\hline \multirow[t]{7}{*}{$2^{\text {nd }}$ Period } & 5 & 22 & 的 & 12 & 1 & 5 & F & 3 & $\mathbf{v}$ & 1 & 4 & 1 & 88 & 1 \\
\hline & & 22 & c. & 12 & 2 & 5 & $\ddot{\theta}$ & 3 & 䚡 & 1 & 2 & 1 & (c) & 1 \\
\hline & $\Rightarrow$ & 22 & $\ldots$ & 11 & 3 & 4 & $\therefore$ & 2 & बखि & 1 & 은 & 1 & 类 & 1 \\
\hline & $\sqrt{ }$ & 22 & ह & 9 & 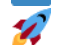 & 4 & 要 & 2 & 8 & 1 & t & 1 & & \\
\hline & & 21 & व' & 8 & $\because$ & 4 & 100 & 2 & $\because$ & 1 & $F$ & 1 & & \\
\hline & 尚 & 17 & 为 & 8 & 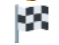 & 4 & 豆 & 2 & $\Leftrightarrow$ & 1 & 4 & 1 & & \\
\hline & 9 & 17 & $i$ & 8 & $y$ & 4 & +8 & 2 & $\ldots$ & 1 & ' & 1 & & \\
\hline
\end{tabular}

Note: Source from twitter.com/Besiktas

As seen in Table 4, when the Beşiktaş JK. Data are considered we find out that 53 different emoji was used in the second period of the 2017-2018 season, 127 in the first period of the 2018-2019 season, and 111 in the second period of the 2018-2019 season. The eagle - $(n=521)$, which is the symbol of the club, emerges as the most used emoji within these periods. Then the flexed biceps $(n=194)$, trophy $\quad(n=148)$, clapping hands $(n=143)$, and geometric shape $\quad(n=109)$ representing the colors of the club were used the most respectively.

Table 5. Content and numbers of emojis in the tweets of Fenerbahçe SK

\begin{tabular}{|c|c|c|c|c|c|c|c|c|c|c|c|c|c|c|}
\hline $\begin{array}{l}\text { Season/ } \\
\text { Period }\end{array}$ & 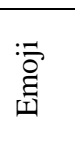 & 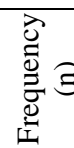 & $\begin{array}{l}:=\overline{0} \\
\text { 产 }\end{array}$ & 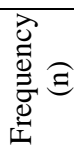 & :- & 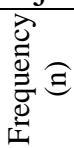 & $\begin{array}{l}:=\sqrt{\circ} \\
\text { 亲 }\end{array}$ & 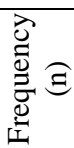 & : & 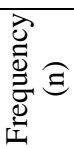 & $\begin{array}{l}: \overline{0} \\
\text { 言 }\end{array}$ & 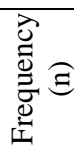 & $\begin{array}{l}:=\bar{\circ} \\
\text { !्ञ }\end{array}$ & 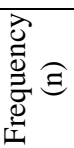 \\
\hline \multirow{9}{*}{$\begin{array}{l}2017-2018 \\
\text { Season } \\
2^{\text {nd }} \text { Period }\end{array}$} & $F$ & 72 & $\frac{11}{21}$ & 21 & 4 & 6 & 橉 & 4 & $M$ & 2 & ! & 1 & $\because$ & 1 \\
\hline & 0 & 61 & VS & 17 & แบข้ & 6 & 2 & 3 & 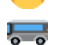 & 2 & $c=5$ & 1 & $M$ & 1 \\
\hline & & 49 & (1) & 13 & InI & 5 & $\mathbb{8}$ & 3 & $\mathrm{e}$ & 2 & 6 & 1 & 8 & 1 \\
\hline & -0 & 44 & 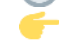 & 13 & It & 5 & , & 3 & 8 & 1 & 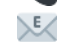 & 1 & (3) & 1 \\
\hline & $\cdots$ & 42 & 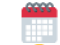 & 12 & (5) & 5 & 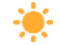 & 3 & 2 & 1 & 4 & 1 & & \\
\hline & 5 & 41 & $\theta$ & 11 & 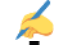 & 5 & 11 & 3 & $\cdots$ & 1 & 6 & 1 & & \\
\hline & 5 & 36 & $\uparrow$ & 9 & ل & 5 & $E$ & 3 & 00 & 1 & ? & 1 & & \\
\hline & (1) & 34 & $\downarrow$ & 9 & $\cdots$ & 4 & D. & 2 & $\theta$ & 1 & 8 & 1 & & \\
\hline & Ð & 27 & C. & 6 & $\mathscr{Q}$ & 4 & ப̆ & 2 & 2 & 1 & (3) & 1 & & \\
\hline \multirow{16}{*}{$\begin{array}{c}2018-2019 \\
\text { Season } \\
1^{\text {st }} \text { Period }\end{array}$} & 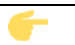 & 135 & $\mathrm{C}$. & $\begin{array}{l}19 \\
\end{array}$ & 4 & 7 & 8 & 4 & 8 & 2 & 3 & 1 & $\square$ & 1 \\
\hline & 0 & 127 & $?$ & 18 & 0 & 7 & 9 & 3 & $\because 7$ & 2 & 84 & 1 & 8 & 1 \\
\hline & $\cdots$ & 83 & Ø̆ & 18 & 랑 & 7 & $\bar{E}$ & 3 & $\because 0$ & 1 & 6 & 1 & (1) & 1 \\
\hline & 8 & 79 & $\bar{T}$ & 17 & $\rho$ & 7 & E & 3 & H. & 1 & $*$ & 1 & $\mathscr{P}$ & 1 \\
\hline & 5 & 75 & \# & 16 & It & 7 & $\Leftrightarrow$ & 3 & i & 1 & 8 & 1 & 8 & 1 \\
\hline & \$" & 48 & $\Leftrightarrow$ & 15 & 8 & 6 & 3 & 3 & $\bigodot_{\text {BACK }}$ & 1 & $\sigma^{\pi}$ & 1 & " & 1 \\
\hline & 6 & 45 & $\overrightarrow{\text { son }}$ & 15 & $\Rightarrow$ & 6 & $\bar{B}$ & 3 & 0 & 1 & ! & 1 & 龄 & 1 \\
\hline & 8 & 33 & VS & 15 & $\theta$ & 6 & $y_{2}^{\prime}$ & 3 & $P$ & 1 & (ن) & 1 & $\hat{\theta}$ & 1 \\
\hline & (1) & 30 & 5 & 13 & 米 & 5 & $E$ & 3 & ! & 1 & 2 & 1 & $\therefore$ & 1 \\
\hline & 0 & 27 & 8 & 12 & 4 & 5 & $' \vec{x} \cdot$ & 3 & ! & 1 & (I) & 1 & d & 1 \\
\hline & แบ๊ & 26 & $\because$ & 11 & 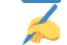 & 5 & 1 & 3 & 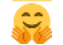 & 1 & (5) & 1 & & \\
\hline & 滆 & 25 & 수 & 10 & 2 & 4 & 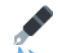 & 3 & ill & 1 & 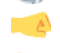 & 1 & & \\
\hline & 彭 & 24 & $\downarrow$ & 10 & 8 & 4 & 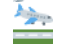 & 2 & $\theta$ & 1 & $(4)$ & 1 & & \\
\hline & 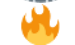 & 23 & 8 & 9 & 80 & 4 & $\equiv$ & 2 & 8 & 1 & $\cdots$ & 1 & & \\
\hline & $\bar{I}$ & 23 & $\sqrt{3}$ & 8 & E & 4 & $\because$ & 2 & $\%$ & 1 & 100 & 1 & & \\
\hline & $\checkmark$ & 21 & 绉 & 8 & 4 & 4 & (1) & 2 & II & 1 & Q & 1 & & \\
\hline \multirow{6}{*}{$\begin{array}{c}2018-2019 \\
\text { Season }\end{array}$} & 5 & 107 & $\overline{V S}$ & 22 & $\Rightarrow$ & 12 & $\square$ & 6 & . & 3 & 西 & 1 & 63 & 1 \\
\hline & 0 & 67 & $?$ & 22 & 5 & 10 & 焉 & 5 & $\overline{1}$ & 3 & $!$ & 1 & 1007 & 1 \\
\hline & 8 & 52 & $\underset{\text { SoON }}{\Rightarrow}$ & 22 & 1 & 10 & 8 & 5 & Hete & 3 & B & 1 & 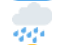 & 1 \\
\hline & $\cdots$ & 46 & (1) & 19 & e & 9 & $\therefore$ & 5 & c. & 2 & $\bar{r}$ & 1 & : & 1 \\
\hline & 6 & 35 & 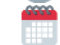 & 19 & 2 & 9 & $\mathscr{Q}$ & 4 & $\%$ & 2 & 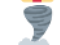 & 1 & DE- & 1 \\
\hline & $\vec{B}$ & 37 & $\checkmark$ & 19 & (1) & 9 & $\Leftrightarrow$ & 4 & (5) & 2 & d & 1 & 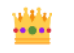 & 1 \\
\hline
\end{tabular}

Jurnal The Messenger, Vol. 13, No. 1 (2021), pp. 63-80 


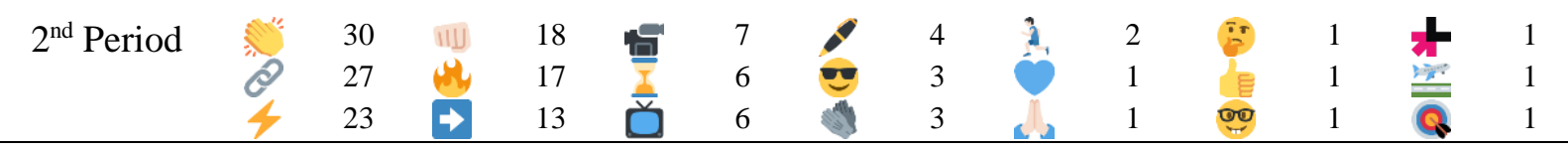

Note: Source from twitter.com/Fenerbahce

As seen in Table 5, Fenerbahçe SK used 58 different emoji in the second period of the 2017-2018 season, 106 in the first period of the 2018-2019 season and 63 in the second period of the 2018-2019 season. Of these emojis, backhand index pointing right $-(n=314)$ and then camera with flash $(n=223)$, speech balloon $\cdots(n=171)$, hearts $00(n=111)$ representing the color of the club and paper clip $\mathcal{O}(n=79)$ are the most commonly preferred emojis.

Table 6. Content and numbers of emojis in the tweets of Galatasaray SK

\begin{tabular}{|c|c|c|c|c|c|c|c|c|c|c|c|c|c|c|}
\hline $\begin{array}{l}\text { Season/ } \\
\text { Period }\end{array}$ & $\begin{array}{l}: \bar{\circ} \\
\stackrel{5}{\square}\end{array}$ & 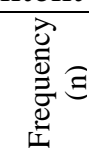 & $\begin{array}{l}: \frac{-}{\circ} \\
\text { !्ञ }\end{array}$ & 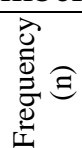 & $\begin{array}{l}:=\frac{\sigma}{\circ} \\
\frac{1}{4}\end{array}$ & 密 & $\begin{array}{l}: \overline{0} \\
\stackrel{\overline{1}}{1}\end{array}$ & 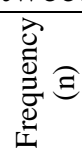 & $\begin{array}{l}: \overline{0} \\
\overline{\mathbf{1}}\end{array}$ & 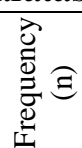 & $\begin{array}{l}: \overline{0} \\
\overline{5} \\
\text { 至 }\end{array}$ & 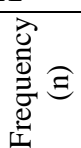 & $\begin{array}{l}: \overline{0} \\
\overline{!} \\
\text { I }\end{array}$ & 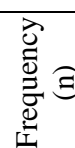 \\
\hline & $\bar{F}$ & 225 & $\cdots$ & 23 & 需 & 15 & the & 7 & 敛 & 3 & हृ & 1 & 0 & 1 \\
\hline & 5 & 198 & (1) & 22 & $\overline{9}$ & 11 & Q & 6 & 2 & 3 & 踏 & 1 & ? & 1 \\
\hline & II & 57 & 吕 & 22 & ק & 10 & $=$ & 6 & 1 & 2 & (1) & 1 & 100 & 1 \\
\hline & 71 & 37 & $\bar{\square}$ & 19 & $!$ & 10 & $\frac{\cos }{21}$ & 6 & 11 & 2 & $\because 7$ & 1 & 1 & 1 \\
\hline 2017-2018 & $\theta$ & 36 & 踾 & 18 & 0 & 10 & VS & 5 & $\frac{z_{n}}{m_{n}}$ & 2 & X & 1 & 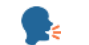 & 1 \\
\hline Season & (1) & 35 & H. & 17 & c. & 9 & แบิ & 5 & E & 2 & dota & 1 & |⿰冫欠 & 1 \\
\hline \multirow{11}{*}{$2^{\text {nd }}$ Period } & (M) & 32 & 急 & 16 & 㝵 & 9 & 量 & 3 & 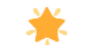 & 2 & $\therefore$ & 1 & 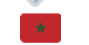 & 1 \\
\hline & 5 & 27 & e & 16 & $\Omega$ & 7 & $=$ & 3 & $\checkmark$ & 1 & 0 & 1 & 0 & 1 \\
\hline & 5 & 26 & S & 15 & $\sqrt{a}$ & 7 & 浃 & 3 & 8 & 1 & 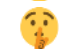 & 1 & $\frac{\xi}{6}$ & 1 \\
\hline & 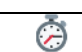 & 257 & 8 & 28 & 9 & 6 & ㅁ & 3 & 0 & 2 & $E$ & 1 & $\theta$ & 1 \\
\hline & 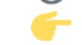 & 246 & \# & 23 & แiो) & 6 & 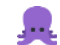 & 3 & 象 & 2 & ב & 1 & 5 & 1 \\
\hline & (2) & 239 & $\Rightarrow$ & 22 & ○ & 5 & 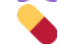 & 2 & 9 & 2 & !! & 1 & 9 & 1 \\
\hline & ह & 145 & 急 & 18 & 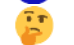 & 5 & (i) & 2 & 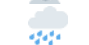 & 2 & $\because$ & 1 & 两 & 1 \\
\hline & ... & 72 & Ø̆ & 17 & $=$ & 5 & 4 & 2 & (द) & 2 & m & 1 & 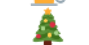 & 1 \\
\hline & 6 & 56 & 8 & 16 & to & 5 & 39 & 2 & $t$ & 2 & 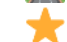 & 1 & $\dot{0}$ & 1 \\
\hline & 3 & 48 & $\infty$ & 13 & 临 & 5 & 8 & 2 & 0 & 2 & ! & 1 & 1 & 1 \\
\hline & 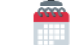 & 46 & \#.". & 11 & 0 & 5 & 乘 & 2 & $\theta$ & 1 & $\varepsilon$ & 1 & 21 & 1 \\
\hline \multirow{15}{*}{$\begin{array}{c}2018-2019 \\
\text { Season } \\
1^{\text {st }} \text { Period }\end{array}$} & है" & 44 & 8 & 10 & $\Leftrightarrow$ & 4 & Q & 2 & $\mathscr{2}$ & 1 & c & 1 & $4 \cdots$ & 1 \\
\hline & 峦 & 40 & की & 9 & & 4 & 3 & 2 & 是 & 1 & $\sum_{\Delta}$ & 1 & 25 & 1 \\
\hline & II & 37 & At & 9 & 1 & 4 & 2 & 2 & $\Leftrightarrow$ & 1 & 4 & 1 & $5 \sqrt{5}$ & 1 \\
\hline & $M$ & 36 & 然 & 9 & AB & 4 & 1 & 2 & 4 & 1 & 6 & 1 & $4 \sqrt[3]{3}$ & 1 \\
\hline & 5 & 36 & $\pi$ & 8 & 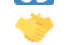 & 3 & $Y_{1}^{\prime}$ & 2 & $\ddot{\theta}$ & 1 & ? & 1 & 89 & 1 \\
\hline & 11 & 36 & i & 7 & 㠯 & 3 & $x$ & 2 & Q & 1 & $\dot{\bar{a}}$ & 1 & & \\
\hline & 5 & 34 & c. & 7 & $=$ & 3 & $\theta$ & 2 & 3 & 1 & - & 1 & & \\
\hline & VS & 33 & $\sqrt{ }$ & 6 & $d$ & 3 & $\because$ & 2 & END & 1 & 0 & 1 & & \\
\hline & (1) & 29 & (D) & 6 & $\Omega$ & 3 & 2 & 2 & 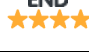 & 1 & 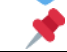 & 1 & & \\
\hline & 8 & 356 & 誉 & 30 & $\vec{c}$ & 10 & (5) & 5 & 3 & 2 & 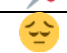 & 1 & $\Delta$ & 1 \\
\hline & 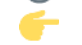 & 253 & $M$ & 24 & 变 & 9 & 0 & 4 & 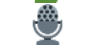 & 2 & ב̌ & 1 & 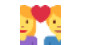 & 1 \\
\hline & 0 & 237 & 尚 & 24 & $\pi$ & 8 & $\ldots$ & 4 & $\theta$ & 2 & !! & 1 & E & 1 \\
\hline & $\ldots$ & 126 & 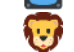 & 23 & 8 & 8 & ? & 4 & 需 & 2 & $\ddot{8}$ & 1 & 8 & 1 \\
\hline & $\theta$ & 107 & VS & 23 & (a) & 8 & 齐 & 3 & $\delta$ & 2 & वे & 1 & $(1)$ & 1 \\
\hline & 5 & 94 & (1) & 23 & 9 & 7 & 管 & 3 & $\Omega$ & 2 & $\because$ & 1 & $\bar{I}$ & 1 \\
\hline \multirow{7}{*}{$\begin{array}{c}2018-2019 \\
\text { Season } \\
2^{\text {nd }} \text { Period }\end{array}$} & 5 & 91 & \# & 23 & 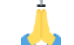 & 7 & $\theta$ & 3 & 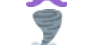 & 2 & 橉 & 1 & $\overline{4}$ & 1 \\
\hline & 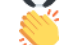 & 72 & $\Rightarrow$ & 22 & 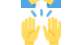 & 7 & $\bar{x}$ & 3 & 100 & 2 & 0 & 1 & $\ddot{\theta}$ & 1 \\
\hline & $\pi$ & 70 & 4 & 18 & 1 & 6 & 2 & 3 & 0 & 2 & 2 & 1 & 60 & 1 \\
\hline & II & 56 & 0 & 15 & $!$ & 6 & 2 & 3 & б.j" & 2 & $\sqrt{11}$ & 1 & Q & 1 \\
\hline & 監 & 56 & (4) & 14 & 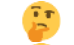 & 5 & 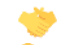 & 2 & 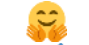 & 1 & . & 1 & $\Leftrightarrow$ & 1 \\
\hline & $\Delta$ & 55 & แบ๊ & 13 & $\theta$ & 5 & 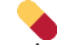 & 2 & $\alpha$ & 1 & 8 & 1 & $?$ & 1 \\
\hline & $T$ & 51 & 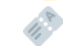 & 12 & Htt & 5 & $\widehat{\circ}$ & 2 & 食 & 1 & है & 1 & & \\
\hline
\end{tabular}




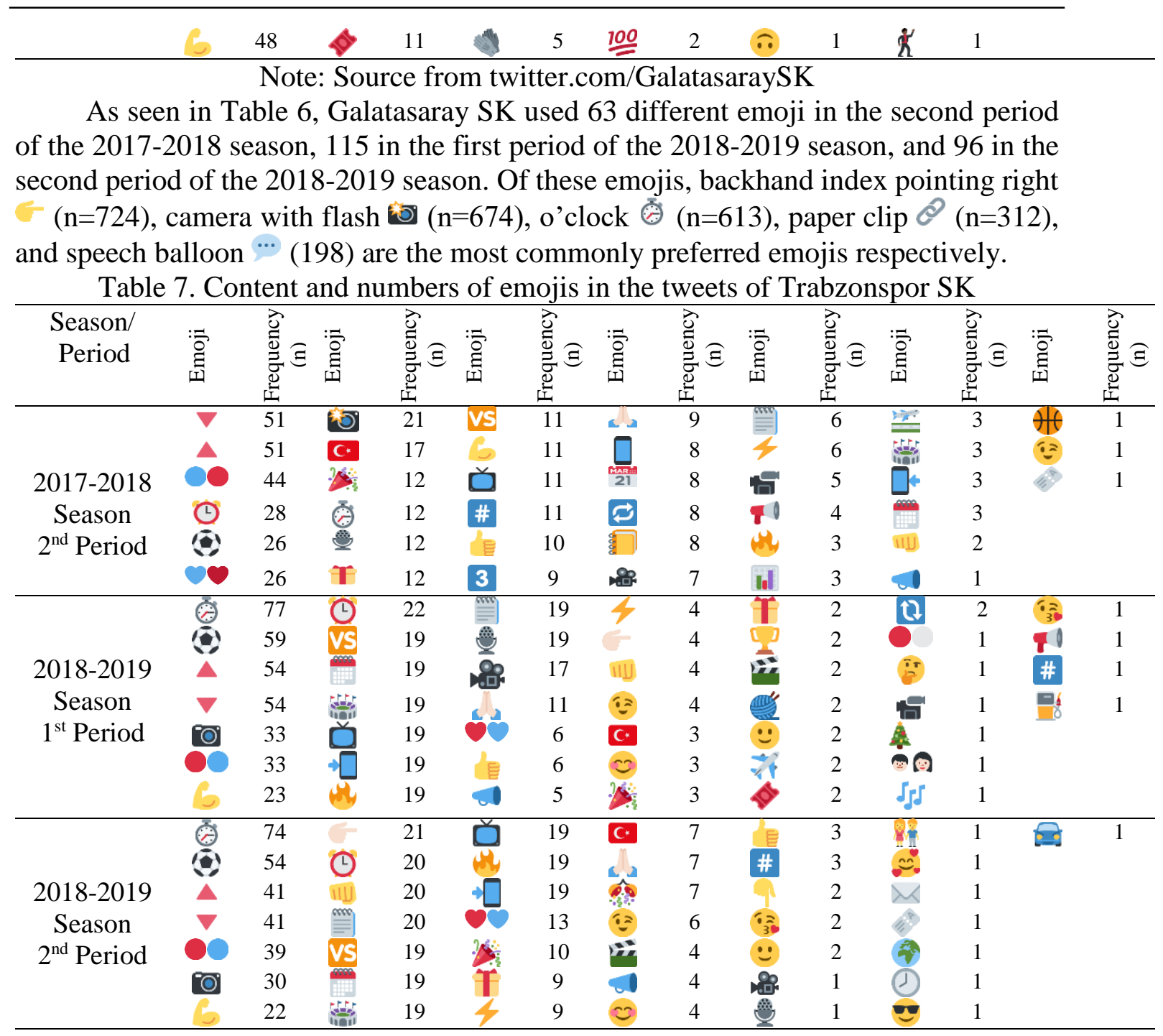

Note: Source from twitter.com/Trabzonspor

As seen in Table 7, Trabzonspor SK used 39 different emoji in the second period of the 2017-2018 season, 46 in the first period of the 2018-2019 season, and 43 in the second period of the 2018-2019 season. Within these periods, the emojis, which are preferred the most, are o'clock $(n=151)$, red triangle pointed down $\nabla(n=146)$, red triangle pointed down $\Delta(n=146)$, geometric shape $0(n=83)$ representing the colors of the club and soccer ball $(\mathrm{n}=81)$ respectively.

\section{Discussion}

Emojis have a crucial role in the everyday communication that we create using tools such as smartphones, tablets, and notebooks (Giannoulis \& Wilde, 2019). The fact that 722 emoji sets were officially added to the Unicode Standard 5.2 for the first time in 2009 has triggered an increase in emoji usage in the social media world. By 2018, more than 2.700 emojis had been added to Unicode Standard 11.0, and emoji use increased day by day in parallel with the rapid change of the communication ways on social networks (Alshenqeeti, 2016; Barbieri et al., 2016). Bosch Jover \& Revilla, (2018) states that nearly six billion emoji are used every day on social media. According to a recent study (Emogi Research Team, 2016), almost everyone who is online (92\% of the online population) uses emoji. The findings of the study are parallel with these 
results. The average of the number of emojis that the sports clubs have used per tweet over the years has increased as seen in Table 2 and reached an average of $1.00(\bar{x}=1.00)$ in the second period of the 2018-2019 season.

It is determined that the emojis within the shares examined within the scope of the study vary depending on the clubs. Considering the study findings, it is seen that Galatasaray SK is the team that posted the most tweets. Then, Besiktas SK, Fenerbahçe SK, Trabzonspor SK and Başakşehir FK are ranked respectively. Many social media platforms impose character restrictions on users when sharing a certain opinion. One of these social media platforms, Twitter limits its users to just 280 characters to express themselves. In this case, the fact that emojis are able to have the meaning of a sentence beyond words in some usages, increase the impact of emotions and thoughts, and make those which are intended to be expressed more powerful has caused users to prefer emojis more frequently (Jaeger, S. R., \& Ares, 2017; Li et al., 2020). It is found that at times the clubs want to increase the impact of emotions and thoughts that are meant to be conveyed by using multiple emoji in one tweet. Accordingly, the fact that Başakşehir FK, which uses the most emoji in its shares despite posting the least number of tweets, uses geometric shapes that symbolize the colors of the club can be interpreted as the club's desire to become a brand through the breakthroughs and successes it has made in recent years.

Similarly, Trabzonspor SK heavily used geometric shape emojis symbolizing the club's colors. National and international sports clubs integrate their assets and brands with their colors (Derbaix \& Decrop, 2011). Therefore, the fact that Başakşehir FK and Trabzonspor SK use the emojis intensively can be evaluated in this context. When the study data is examined, it is determined that Başakşehir FK, Galatasaray SK, and Fenerbahçe SK used camera emojis intensively. It can be stated that the relevant teams use camera emoji heavily in training images and photos related to the joys of goals in live matches. According to the Instagram Industry Report by Simply Measured (Jackson, 2015), brands use camera emojis the most. Accordingly, the use of the camera by sports clubs reveals that fan interaction is observed in a professional manner.

When the data of the study are evaluated, it is seen that the sports clubs use emojis that identify themselves in the tweets they make. For example, it is seen that Galatasaray SK uses 'crown' and 'lion' emojis more than other emojis. Each of these posts bears different meanings. Accordingly, the 'lion' emoji refers to a lion which is the symbol of Galatasaray SK, while the 'crown' emoji bears the meanings regarding the qualifying lion as the king of the forests. Likewise, it is determined that Besiktas JK intensively shared the 'eagle' emoji and the 'trophy' emoji. Since all of these posts bear the histories and symbols of the teams, they can be regarded as the attempts of the teams to establish a warm and exciting communication with the fans.

The studies (Ganster et al., 2012; Rodrigues et al., 2018) show that recipients attribute greater sentimentality to emoji-added messages and that emojis make people feel good (Das et al., 2019; Hill, 2016). In this respect, it can be stated that the use of emoji in social media messages by sports clubs examined in the scope of the study allows them to establish more sentimental communication with fans. Also, some studies on emojis (Jaeger et al., 2018; Tian et al., 2017) indicates that the emojis that create positive connotations, rather than the ones that create negative connotations, are used in social media shares. The fact that the sports clubs use the emojis that create positive and neutral connotations in all of their tweets examined within the scope of the study supports this result. 
The digital structure of new media brings about options such as interactivity and user-generated content (Lefever, 2012). Its interactive structure and user-controlled content have caused the boundaries between the new media and the sports sector which have similar characteristics to become ambiguous and intertwined. Accordingly, emojis, which gradually increase their position in everyday life, attract the attention of sports clubs due to the influence of popular culture and are used in many communication studies. While emojis shared by sports clubs are adopted by fans and consumers as part of their own culture, this situation is regarded as an element of addressing their emotions (Kaye., 2015). As they are evaluated from a different point of view, it is possible to say that emojis are a kind of promotional activity. In this context, clubs using emoji want to differentiate themselves from their competitors and create a common language with them.

The findings of this study have to be seen in light of some limitations. The emojis examined in this study were separated from the tweet texts and counted one by one, and some inferences were made from the total emojis. Therefore, very rarely, the meanings of these emojis in their context may differ. The present study examined the emoji usage patterns and frequency of sports clubs in Turkey specific to Twitter and the data were collected from Turkish sport clubs only. While the data provided important contribution for integrated sports marketing communication, these findings cannot be generalized to all sports clubs. Also known that emojis has a different meaning by different countries with their cultures. So in future studies, universal qualities of emoji use can be addressed by including various cultural codes, increasing the number of clubs to be examined on a national and international basis. In addition, various comparisons can be made among different countries. In addition to this, different comparisons and inferences can be made depending on the social media networks by analyzing the content of the posts on Twitter and other social media networks. All these studies can contribute to the communication between sports clubs and fans-followers, as well as the fields of marketing communication in sports and strategic sports communication.

\section{Conclusion}

The study found that sports clubs use emojis that create positive and neutral connotations. Emojis used are heavily determined to be visuals depicting the colors and symbols of sports clubs. However, it was found that the sports clubs that are the sample of the study use emojis in almost all of the posts they make on Twitter. This indicates that the use of emojis in the context of sports clubs' tweets is very important in terms of direct communication with and transmission of emotions to their fans-followers. As a result of the study, it was found that emojis, which were proved that they created a new and common language within the framework of interactive communication with online consumers via Twitter, which can be viewed as one of the main goals of sports clubs, are very important and necessary for sports clubs in their marketing communication works, in creating loyalty, as well as reflecting the feelings and thoughts that are intended to be conveyed on individuals more effectively.

\section{Conflict of Interest}

There is no conflict of interest with any financial, personal, or other relationships with other people or organization related to the material discussed in the manuscript.

Jurnal The Messenger, Vol. 13, No. 1 (2021), pp. 63-80 


\section{Acknowledgements}

The Research Team would like to thank the all sports clubs to give permission to examined their Twitter accounts and tweets.

\section{References}

Abeza, G., O’Reilly, N., Seguin, B., \& Nzindukiyimana, O. (2017). Social media as a relationship marketing tool in professional sport: A netnographical exploration. International Journal of Sport Communication, 10(3), 325-358. https://doi.org/https://doi.org/10.1123/ijsc.2017-0041

Alshenqeeti, H. (2016). Are emojis creating a new or old visual language for new generations? A socio-semiotic study. Advances in Language and Literary Studies, 7(6), 56-69. https://doi.org/https://doi.org/10.7575/aiac.alls.v.7n.6p.56

Barbieri, F., Ronzano, F., \& Saggion, H. (2016). What does this emoji mean? A vector space skip-gram model for twitter emojis. In. In N. Calzolari, K. Choukri, T. Declerck, S. Goggi, M. Grobelnik, B. Maegaard, J. Mariani, H. Mazo, A. Moreno, J. Odijk, \& S. Piperidis. (Eds.), Proceedings of the Tenth International Conference on Language Resources and Evaluation (LREC 2016) (pp. 3967-3972). European Language Resources Association. http://www.lrecconf.org/proceedings/lrec2016/summaries/735.html

Bauer, H., Barnes, S. J., Reichardt, T., \& Neumann, M. . (2005). Driving consumer acceptance of mobile marketing: A theoretical framework and empirical study. Journal of Electronic Commerce Research, 6(3), 181-192. http://directory.umm.ac.id/articles/Baueretal_MomMarketingConsumerAccept.p $\mathrm{df}$

Berard, B. (2018). I second that emoji: The standards, structures, and social production of emoji. First Monday, https://doi.org/https://doi.org/10.5210/fm.v23i9.9381

Blagdon, J. (2013). How emoji conquered the World. Theverge. https://www.theverge.com/2013/3/4/3966140/how-emoji-conquered-the-world

Blaszka, M. (2011). An xamination of sport consumers' Twitter usage [Unpublished master of science dissertation] [Georgia State University]. https://scholarworks.gsu.edu/kin_health_theses/1

Bosch Jover, O., \& Revilla, M. A. (2018). The Use of Emojis by Millennials (No. 57; RECSM Working Paper). https://www.upf.edu/documents/3966940/6839730/Working+Paper_Emoji_Subs tantive.pdf/bbbf386b-864f-3116-b8fb-dece14760a45

Boulianne, S. (2015). Social media use and participation: A meta-analysis of current research. Information, Communication \& Society, 18(5), 524-538. https://doi.org/https://doi.org/10.1080/1369118X.2015.1008542

Cincu, R. (2017). Emoticons: between Linguistic innovation and Symptom of the Posthuman Era. Journal of Media Research-Revista de Studii Media, 10(27), 8895.

Cowie, R., Douglas-Cowie, E., Tsapatsoulis, N., Votsis, G., Kollias, S., Fellenz, W., \& Taylor, J. G. (2001). Emotion recognition in human-computer interaction. IEEE Signal Processing Magazine, 18, 32-80. https://doi.org/10.1109/79.911197.

Danesi, M. (2017). The semiotics of emoji: The rise of visual language in the age of the internet. Media and Communication, 5(4), 75. https://doi.org/10.17645/mac.v5i4.1041 
Das, G., Wiener, H. J., \& Kareklas, I. (2019). To emoji or not to emoji? Examining the influence of emoji on consumer reactions to advertising. Journal of Business Research, 96

147-156. https://doi.org/https://doi.org/10.1016/j.jbusres.2018.11.007

Derbaix, C., \& Decrop, A. (2011). Colours and scarves: an ethnographic account of football fans and their paraphernalia. Leisure Studies, 30(3), 271-291. https://doi.org/https://doi.org/10.1080/02614367.2010.527356

Dima, T. (2015). Social media usage in European clubs football industry. Is digital reach better correlated with sports or financial performance? The Romanian Economic Journal, 18(55), 117-128. https://ideas.repec.org/a/rej/journl/v18y2015i55p117128.html

Dimson, T. (2015). Emojineering Part 1: Machine Learning for Emoji Trends. Instagram Engineering. https://instagram-engineering.com/emojineering-part-1machine-learning-for-emoji-trendsmachine-learning-for-emoji-trends7f5f9cb979ad

Drapier, E. P., \& Tezonarici, S. D. (2019). Analysis of Twitter behaviors of Portuguese and Turkish clubs [Molde University College]. https://himolde.brage.unit.no/himoldexmlui/bitstream/handle/11250/2620824/master_perdigao_drapieri.pdf?sequence $=1 \&$ isAllowed $=\mathrm{y}$

Dresner, E., \& Herring, S. C. (2010). Functions of the nonverbal in CMC: Emoticons and illocutionary force. Communication Theory, 20(3), 249-268. https://doi.org/https://doi.org/10.1111/j.1468-2885.2010.01362.x

Dua, T. (2015). The emojification of brand advertising. Digiday. https://digiday.com/marketing/emojification-brand-advertising/

Emogi Research Team. (2016). Emoji Report. https://cdn.emogi.com/docs/reports/2016_emoji_report.pdf

Fahlman, S. E. (2002). Smiley Lore:-). Carnegie Mellon University. https://www.cs.cmu.edu/ sef/sefSmiley.htm

Figge, S. (2004). Situation-dependent services-a challenge for mobile network operators. Journal of Business Research, 57(12), 1416-1422. https://doi.org/https://doi.org/10.1016/S0148-2963(02)00431-9

Filo, K., Lock, D., \& Karg, A. (2015). Sport and social media research: A review. Sport Management Review, 18(2), 166-181. https://doi.org/https://doi.org/10.1016/j.smr.2014.11.001

Fraenkel, J. R., Wallen, N. E., \& Hyun, H. H. (2012). How to Design and Evaluate Research in Education (8th ed.). McGraw-Hill Companies, Inc.

Ganster, T., Eimler, S. C., \& Kraemer, N. C. (2012). Same same but different!? The differential influence of smiles and emoticons on person perception. Cyberpsychology, Behavior, and Social Networking, 15(4), 226-230. https://doi.org/10.1089/cyber.2011.0179.

Garris, M., \& Mishra, K. (2014). A beginner's guide to mobile marketing. Business Expert Press.

Giannoulis, E., \& Wilde, L. R. A. (2019). Emoticons, kaomoji, and emoji: The transformation of communication in the digital age (1st ed.). Routledge.

Gibbs, C., \& Haynes, R. (2013). A phenomenological investigation into how Twitter has changed the nature of sport media relations. International Journal of Sport Communication,

$7(2)$

$188-213$. 
https://doi.org/https://doi.org/10.1123/IJSC.2014-0005

Gibbs, C., O'Reilly, N., \& Brunette, M. (2014). Professional Team Sport and Twitter: Gratifications Sought and Obtained by Followers. International Journal of Sport Communication, $7(2)$

$188-213$. https://doi.org/https://doi.org/10.1123/IJSC.2014-0005

Grewal, D., \& Levy, M. (2019). Marketing. (6th ed.). McGraw-Hill.

Grönroos, C. (2004). The relationship marketing process: Communication, interaction, dialogue, value. Journal of Business \& Industrial Marketing, 19(2), 99-113. https://doi.org/https://doi.org/10.1108/08858620410523981

Gülşen, T. T. (2016). You tell me in emojis. In T. Ogata \& T. Akimoto (Eds.), Computational and Cognitive approaches to Narratology (pp. 354-375). IGI Global. https://doi.org/10.4018/978-1-5225-0432-0.ch014

Hambrick, M. E. (2012). Six degrees of information: Using social network analysis to explore the spread of information within sport social networks. International Journal of Sport Communication, 5(1), 16-34. https://doi.org/https://doi.org/10.1123/ijsc.5.1.16

Highfield, T. (2018). Emoji hashtags//hashtag emoji: of platforms, visual affect, and discursive flexibility. First Monday, 23(9). https://doi.org/https://doi.org/10.5210/fm.v23i9.9398

Hill, J. H. (2016). The impact of emojis and emoticons on online consumer reviews, perceived company response quality, brand relationship, and purchase intent [Unpublished master of arts dissertation [University of South Florida]. https://digitalcommons.usf.edu/etd/6513

Hopkins, J. L. (2013). Engaging Australian rules football fans with social media: A case study. International Journal of Sport Management and Marketing, 13(1/2), 104121. https://doi.org/10.1504/IJSMM.2013.055197

Hougaard, T. T., \& Rathje, M. (2018). Emojis in the digital writings of young Danes. In A. Ziegler (Ed.), Jugendsprachen/Youth languages: Aktuelle perspektiven internationaler forschung/current perspectives of international research (pp. 773-806). De Gruyter. https://doi.org/https://doi.org/10.1515/9783110472226

Jackson, D. (2015). How to Go Viral: 8 Steps to Reach a Massive Audience. Sproutsocial. https://sproutsocial.com/insights/how-to-go-viral/

Jaeger, S. R., \& Ares, G. (2017). Dominant meanings of facial emoji: Insights from Chinese consumers and comparison with meanings from internet resources. Food Quality and Preference, 62, 275-283. https://doi.org/https://doi.org/10.1016/j.foodqual.2017.04.009

Jaeger, S. R., Vidal, L., Kam, K., \& Ares, G. (2018). Can emoji be used as a direct method to measure emotional associations to food names? Preliminary investigations with consumers in USA and China. Food Quality and Preference, 68, 38-48. https://doi.org/https://doi.org/10.1016/j.foodqual.2016.09.005

Kaye., K. (2015). FORD, DOMINO'S, DELL, OTHERS WORK TO TRANSLATE THE LANGUAGE OF EMOJIS. Advertising Age. https://adage.com/article/digital/marketers-work-translate-languageemojis/301064

Kolpe, R., \& Burnett, M. (1991). Content analysis research: An examination of applications with directives for improving research reliability and objectivity. Journal of Consumer Research, 18(2), 243-250. https://doi.org/https://doi.org/10.1086/209256 
Krippendorff, K. (2004). Reliability in Content Analysis: Some Common Misconceptions and Recommendations. Human Communication Research, 30(3), 411-433. https://doi.org/https://doi.org/10.1111/j.1468-2958.2004.tb00738.x

Lefever, K. (2012). New Media and Sport. Springer. https://link.springer.com/book/10.1007/978-90-6704-873-6

Li, J., Longinos, G., Wilson, S., \& Magdy, W. (2020). Emoji and Self-Identity in Twitter Bios. Proceedings of the Fourth Workshop on Natural Language Processing and Computational Social Science. https://doi.org/http://dx.doi.org/10.18653/v1/2020.nlpcss-1.22

Lin, K.-Y., \& Lu, H.-P. (2011). Intention to Continue Using Facebook Fan Pages from the Perspective of Social Capital Theory. Cyberpsychology, Behavior, and Social Networking, 14(10). https://doi.org/https://doi.org/10.1089/cyber.2010.0472

Lombard, M., Snyder-Duch, J., \& Bracken, C. C. (2006). Content Analysis in Mass Communication: Assessment and Reporting of Intercoder Reliability. Human Communication Research, 28(4), 587-604. https://doi.org/https://doi.org/10.1111/j.1468-2958.2002.tb00826.x

M.Nisar, T., Prabhakar, G., \& P.Patil, P. (2018). Sports clubs' use of social media to increase spectator interest. International Journal of Information Management, 43, 188-195. https://doi.org/https://doi.org/10.1016/j.ijinfomgt.2018.08.003

Manovich, L. (2001). The Language of New Media. The MIT Press.

Maree, T. (2017). The Social Media Use Integration Scale: Toward Reliability and Validity. International Journal of Human-Computer Interaction, 33(12), 963972. https://doi.org/https://doi.org/10.1080/10447318.2017.1301041

Marengo, D., Giannotta, F., \& Settanni, M. (2017). Assessing personality using emoji: An exploratory study. Personality and Individual Differences, 112, 74-78. https://doi.org/https://doi.org/10.1016/j.paid.2017.02.037

McBain, S. (2017). Happy face, sad face - are emojis the nearest thing to a universal language? More people "speak" emoji than English. New Statesman. https://www.newstatesman.com/science-tech/2017/09/happy-face-sad-face-areemojis-nearest-thing-universal-language

Neuman, W. L. (2020). Social research methods: Qualitative and quantitative approaches (8th ed.). Pearson.

Nicholson, M., Kerr, A., \& Sherwood, M. (2015). Sport and the Media: Managing the Nexus. (2nd ed.). Routledge. https://www.routledge.com/Sport-and-the-MediaManaging-the-Nexus/Nicholson-Kerr-Sherwood/p/book/9780415839822

Novak, P. K., Smailović, J., Sluban, B., \& Mozetič, I. (2015). Sentiment of Emojis. PLoS One, 1-22. https://doi.org/10.1371/journal.pone.0144296

O'Shea;, M., \& Alonso, A. D. (2011). Opportunity or obstacle? A preliminary study of professional sport organisations in the age of social media. International Journal of Sport Management and Marketing, 10(3/4), 196-212. https://doi.org/https://dx.doi.org/10.1504/IJSMM.2011.044790

Oelrichs, I. (2020). Adoption of Innovations in Digital Sports Journalism: The Use of Twitter by German Sports Journalists. Sage Journals. https://doi.org/https://doi.org/10.1177\%2F2167479520961786

Pavalanathan, U., \& Eisenstein, J. (2016). More emojis, less :) The competition for paralinguistic function in microblog writing. First Monday, 21(11). https://doi.org/https://doi.org/10.5210/fm.v21i11.6879

Pegoraro, A. (2010). Look Who's Talking-Athletes on Twitter: A Case Study. 
International Journal of Sport Communication, 3, 501-514. https://doi.org/https://doi.org/10.1123/ijsc.3.4.501

Pohl, H., Domin, C., \& Rohs, M. (2017). Beyond Just Text: Semantic Emoji Similarity Modeling to Support Expressive Communication ??? ACM Transactions on Computer-Human Interaction, 24(1), 1-42. https://doi.org/https://doi.org/10.1145/3039685

Poster, M. (2013). The Second Media Age. Polity. https://www.wiley.com/enus/The+Second+Media+Age-p-9780745668239

Potter, W. J., \& Levine-Donnerstein, D. (2009). Rethinking Validity and Reliability in Content Analysis. Journal of Applied Communication Research, 27(3), 258-284. https://doi.org/https://doi.org/10.1080/00909889909365539

Price, J., Farrington, N., \& Hall, L. (2013). Changing the game? The impact of Twitter on relationships between football clubs, supporters and the sports media. Soccer \& Society, 14(4), 446-461. https://doi.org/https://doi.org/10.1080/14660970.2013.810431

Rezabek, L., \& Cochenour, J. (1998). Visual Cues in Computer-Mediated Communication: Supplementing Text with Emoticons. Journal of Visual Literacy, 18(2), 201-215. https://doi.org/https://doi.org/10.1080/23796529.1998.11674539

Rodrigues, D., Prada, M., Gaspar, R., Garrido, M. V., \& Lopes, D. (2018). Lisbon Emoji and Emoticon Database (LEED): Norms for emoji and emoticons in seven evaluative dimensions. Behav Res, 50(392-405). https://doi.org/https://doi.org/10.3758/s13428-017-0878-6

Selken, N. (2019). Emoji Dictionary. Moji Dictionary. https://emojidictionary.emojifoundation.com/

Siguencia, L. O., Marzano, D. H. G., \& Rodak, P. (2017). The Role of Social Media in Sports Communication Management: An Analysis of Polish Top League Teams' Strategy. Procedia Computer Science, 104, 73-80. https://doi.org/https://doi.org/10.1016/j.procs.2017.01.074

Stark, L., \& Crawford, K. (2015). The Conservatism of Emoji: Work, Affect, and Communication. Sage Journals. https://doi.org/https://doi.org/10.1177\%2F2056305115604853

Swaney-Stueve, M., Jepsen, T., \& Deubler, G. (2018). The emoji scale: A facial scale for the 21st century. Food Quality and Preference, 68, 183-190. https://doi.org/https://doi.org/10.1016/j.foodqual.2018.03.002

Tian, Y., Galery, T., Dulcinati, G., Molimpakis, E., \& Sun, C. (2017). Facebook sentiment: Reactions and Emojis. In L.-W. Ku \& C.-T. Li (Eds.), Proceedings of the Fifth International Workshop on Natural Language Processing for Social Media. Association for Computational Linguistics. https://aclanthology.org/W171102

Tocci, R. J. ., Widmer, N., \& Moss, G. (2007). Digital Systems: Principles and Applications (10th ed.). Pearson. https://www.pearson.com/uk/educators/highereducation-educators/program/Tocci-Digital-Systems-Principles-and-

Applications-10th-Edition/PGM789304.html

Tweetfc. (2019). Tweetfc. Tweetfc. http://www.tweetsfc.com/stats/most-followedfootball-teams-on-twitter

Wang, Y. (2021). Building relationships with fans: how sports organizations used twitter as a communication tool. Sport in Society, 24(7). https://doi.org/https://doi.org/10.1080/17430437.2020.1725475 
Wang, Y., \& Zhou, S. (2015). How Do Sports Organizations Use Social Media to Build Relationships? A Content Analysis of NBA Clubs' Twitter Use. International Journal of Sport Communication, 8(2), 133-148. https://doi.org/https://doi.org/10.1123/ijsc.2014-0083

Watkins, B. A. (2013). Social media \& sports: an evaluation of the influence of Twitter and mobile apps on brand-related consequences [University of Alabama]. https://ir.ua.edu/handle/123456789/1821

We are Social Inc. (2019). Digital 2019: Internet trends in 2019. Wearesocial. https://wearesocial.com/global-digital-report-2019

Weber, R. (1990). Basic Content Analysis (2nd ed.). SAGE researchmetode. https://doi.org/https://dx.doi.org/10.4135/9781412983488

Williams, J., \& Chinn, S. J. (2010). Meeting Relationship-Marketing Goals Through Social Media: A Conceptual Model for Sport Marketers. International Journal of Sport Communication, 3(4), 422-437. https://doi.org/https://doi.org/10.1123/ijsc.3.4.422

Winand, M., Belot, M., Merten, S., \& Kolyperas, D. (2019). International Sport Federations' Social Media Communication: A Content Analysis of FIFA's Twitter Account. International Journal of Sport Communication, 12(2), 209-233. https://doi.org/https://doi.org/10.1123/ijsc.2018-0173

Yengin, D. (2015). Yeni medyanın olanakları: Semantik Web. The Turkish Online Journal of Design, Art and Communication, 5(1), 44-53. http://tojdac.org/tojdac/VOLUME5-ISSUE1_files/tojdac_v05i104.pdf

Zanini, M. T., Moraes, F. C. de, Lima, V., Migueles, C., Lourenco, C., \& Irigaray, H. A. R. (2019). Soccer and Twitter: virtual brand community engagement practices. Marketing Intelligence \& Planning, 37(7), 791-805. https://doi.org/https://doi.org/10.1108/MIP-08-2018-0371

Zhou, R., Hentschel, J., \& Kumar, N. (2017). Goodbye Text, Hello Emoji: Mobile Communication on WeChat in China. Proceedings of the 2017 CHI Conference on Human Factors in Computing Systems, 748-759. https://doi.org/https://doi.org/10.1145/3025453.3025800 